\title{
Modelo de actividades para la caracterización de procesos de toma de decisiones no programadas enmarcadas en escenarios de negociación
}

\author{
Javier Albadán Romero, Paulo Alonso Gaona García \\ Universidad Distrital Francisco José de Caldas, \\ Bogotá, Colombia
}

\begin{abstract}
Resumen. La toma de decisiones (TD) es una trascendental habilidad en cargos de alta dirección organizacional. Allí, diferentes actividades ejercidas pueden ser comprendidas dentro del concepto de negociación, el cual tiene un estrecho vínculo con la TD. La TD no programadas son llevadas a cabo por una persona en escenarios atípicos donde la información es ambigua y conlleva la implementación de métodos de solución poco habituales. Actualmente existen modelos matemáticos que especifican la TD desde diferentes enfoques, no obstante la TD no programadas todavía es un campo de acción por explorar, pues no se han determinado estrategias sólidas que fundamenten procesos de TD en escenarios atípicos. A partir de este panorama, este artículo tiene como propósito presentar un modelo de relación entre actividades que permitan caracterizar la TD no programadas y los escenarios de negociación, con el propósito de ser usado después en un entorno de gamificación aplicando agentes inteligentes.
\end{abstract}

Palabras clave: Toma de decisiones no programadas, alta gerencia, negociación, modelo de caracterización.

\section{Introducción}

Los gerentes de niveles estratégicos y operativos en muchas organizaciones enfrentan, con frecuencia, problemas de decisión similares que pueden variar debido a diferencias en sus estilos de toma de decisiones así como de la subjetividad, tal como se define en [1]. Por su parte, las decisiones tomadas en actividades que se llevan a cabo en la alta dirección son denominadas decisiones estratégicas desde la perspectiva expuesta en [2]. Las decisiones estratégicas se definen como elecciones de tal importancia que puedan comprometer la asignación de determinados recursos, así como el alcance y mantenimiento de la ventaja competitiva en la organización.

Específicamente, la toma de decisiones es un proceso meta cognitivo involucrado en casi todo proceder cotidiano, el cual ocurre durante unos pocos segundos en la mente humana, tanto en acciones conscientes como subconscientes. La toma de decisiones se puede detallar a partir de 3 elementos constituyentes según [3]: la situación de decisión, quién toma las decisiones (negociador) y el proceso de decisión. Desde otra perspectiva, [4] expone tres elementos esenciales para la toma de decisiones: las metas de decisión, un conjunto de elecciones alternativas y un conjunto de criterios o estrategias. 
Existen algunas propuestas orientadas definir modelos de caracterización de procesos en tomas de decisiones, en esa vía [4] llevaron a cabo un estudio sobre la taxonomía de las estrategias y criterios de la toma de decisiones donde lograron establecer 4 categorías que abarcan los 24 criterios presentados. Por su parte [5] exponen los principales modelos matemáticos asociados a los procesos de toma de decisiones, detallando diferentes enfoques orientados a apoyar la toma de decisiones.

En cuanto a la toma de decisiones no programadas, [6] se definen dos tipos de problemas: estructurados y no estructurados y presenta una relación con las mismas categorías de toma de decisiones. En esa vía, recibir información inexacta o perder alternativas puede generar que se tomen decisiones imprecisas como lo explican [7]. Para [8] en una organización las decisiones no son completamente programadas ni completamente no programadas, sino una combinación de ambos tipos. No obstante estas nociones, la toma de decisiones no programadas todavía es un campo de acción por explorar pues no es muy amplio el desarrollo de estudios en esta vía.

A partir de este panorama, la motivación de este artículo se centra en profundizar en el estudio de la toma de decisiones no programadas, con el propósito de generar y especificar un modelo para caracterizar procesos de toma de decisiones no programadas en escenarios de negociación de alta gerencia.

El artículo se encuentra organizado de la siguiente manera: en la sección 2 se presentan las características generales de la toma de decisiones no programadas, sustentando su relación con la alta dirección en organizaciones. En la sección 3 se relacionan los detalles metodológicos. En la sección 4 se propone el modelo que permitirá caracterizar actividades de toma de decisiones no programadas en escenarios de negociación. Finalmente se plantean las conclusiones y trabajos futuros.

\section{Marco teórico}

En esta sección se abordan los aspectos trascendentales de la toma de decisiones en general para luego enfocarse sobre las toma de decisiones no programadas. Posteriormente se sustentará la relación entre la toma de decisiones y la alta gerencia. Con esta base conceptual, se pretende dar sustento para establecer relación con las nociones de negociación en la discusión y concretar así el modelo aquí propuesto.

\subsection{Características cognitivas de la toma de decisiones}

La toma de decisiones es uno de los 37 procesos cognitivos fundamentales que se encuentra en la capa cognitiva superior del cerebro. En este se concentra la elección de una opción de preferencia entre un conjunto de posibilidades tal como se expone en [9], quienes describen un modelo de referencia de capas del cerebro, tal como se ilustra en la Fig. 1. Describen una función cognitiva superior como una función vital avanzada del cerebro que se desarrolla y adquiere con el apoyo de funciones meta cognitivas.

Entre otras funciones del cerebro de esta capa superior, de acuerdo a [10], se encuentran el reconocimiento, la imaginación, comprensión, aprendizaje, razonamiento, deducción, inducción, toma de decisiones, resolución de problemas, la explicación, análisis, síntesis, creación, analogía, planificación y cuantificación. Todas estas funciones se encuentran clasificadas dentro de los procesos conscientes del 
cerebro, los cuales se caracterizan por ser adquiridos, moldeados, se pueden controlar intencionadamente y que se basan en la voluntad, metas y motivaciones.
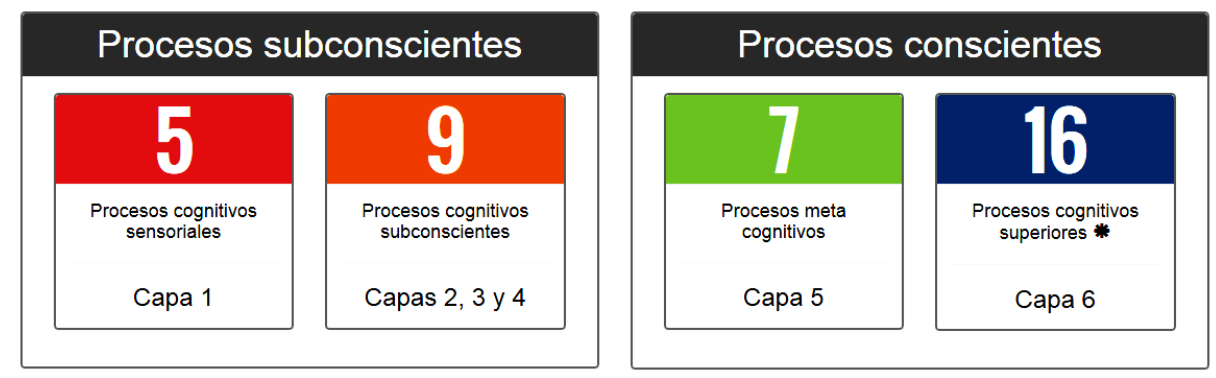

* Toma de Decisiones $\rightarrow$ Capa cognitiva superior del cerebro

Fig. 1. Procesos cognitivos del modelo de referencia de capas del cerebro en Wang et al. [9].

La toma de decisiones está directamente involucrada con la solución de problemas, como comportamiento base de la conducta humana. Se ilustra en [4] que un espacio de problema incluye: 1) la descripción de estados posibles de la tarea y quien va a solucionar el problema y 2) la lista de las formas de moverse entre los estados de búsqueda.

\subsection{Toma de decisiones no programadas}

Los procesos de toma de decisiones son determinados por el tipo de problema que se va a solucionar. En la misma vía en que están definidos los tipos de problemas, la toma de decisiones presenta también dos tipos básicos de clasificación: la toma de decisiones programadas y la toma de decisiones no programadas. Estos dos tipos de toma de decisiones corresponden a problemas estructurados y no estructurados respectivamente. Del primer tipo es esencial mencionar que son decisiones rutinarias o repetitivas, tal como mencionan en [8], siendo en esencia una decisión tomada con base en antecedentes o que se ciñen a criterios prestablecidos o reglas de decisión. Un operario de una máquina o el conductor de un automóvil ejecutan este tipo de decisiones pues las acciones que llevan a cabo ya están interiorizadas.

La toma de decisiones no programadas se aplica en problemas no estructurados, los cuales por su condición de novedad y vaga definición implica recurrir a procesos de solución no frecuentes pues quién va a tomar la decisión no tiene interiorizados ciertos métodos básicos de solución. El problema no estructurado se erige en un escenario excepcional, no habitual, por ende las acciones de respuesta no son instantáneas según Corona [6]. Esta situación puede tener repercusiones ciertamente complejas teniendo en cuenta que en algunos escenarios, se pueden tomar decisiones imprecisas porque se pierden alternativas o se recibe información inexacta sobre los posibles resultados de las diferentes opciones como se explica en [7].

Finalmente, en [8] resaltan que las decisiones en una organización no son completamente programadas ni completamente no programadas, sino una combinación de ambos tipos, lo cual debe ser dominado de un experto en toma de decisiones. 


\subsection{Toma de decisiones en la alta dirección}

En muchas organizaciones, los gerentes de niveles estratégicos y operativos se ven enfrentados con frecuencia a problemas de decisión con condiciones similares, en donde se pueden tomar diferentes rumbos debido a diferencias en sus estilos de toma de decisiones y a la subjetividad, como se sustenta en [1]. Las decisiones tomadas en actividades que se llevan a cabo en la alta dirección son denominadas decisiones estratégicas [2]. Estas decisiones estratégicas presentan gran importancia en tanto comprometen la asignación de determinados recursos, así como el alcance y mantenimiento de la ventaja competitiva en la organización. Para [11] la toma de decisiones estratégicas representa un elemento clave en la alta dirección en la medida en que determina una alineación de los recursos y capacidades, de la organización, respecto a las oportunidades y amenazas que existen en el entorno.

Un término que es susceptible de ser atribuido a este contexto de la toma de decisiones en la alta dirección, es el planteado en [12], donde se referencia que un juego gerencial es un modelo matemático, donde a partir de ciertas fórmulas se hace uso de un raciocinio lógico para las decisiones, pero adicionalmente, se considera estocástico porque implica aleatoriedad en tanto existen variables que influyen directamente en el comportamiento de la organización sin que ésta pueda establecer control sobre dichas variables (indicadores económicos y competencia). Lo interesante de abordar esta perspectiva, es que se establece el vínculo directo con la teoría de juegos que se encarga de estudiar la elección ideal cuando las decisiones dependen de los demás involucrados, haciendo uso de argumentos de racionalidad y restricciones presentes.

\subsection{Negociación}

El concepto de negociación se acota teniendo en cuenta el abordaje conceptual de que se plantea en [13] quien la define como un proceso en el que una decisión conjunta se hace por dos o más partes. Las partes deben expresar postulaciones contradictorias para después impulsarse hacia un acuerdo mediante un proceso de "concesión de decisiones" o por la búsqueda por nuevas alternativas. En adición, [14] señalan que la negociación es una actividad que requiere entrenamiento, practica, estrategia y preparación, siendo elementos claves para la ejecución de acuerdos que son aceptados mutuamente por las contrapartes, a pesar de que sucedan diversos conflictos y se requiera ayuda externa.

Con la intención de abordar el uso de agentes computacionales en contextos de negociación, [15] hacen un detalle de las tareas que desarrollaría un agente para llevar a cabo procesos de negociación, los cuales sintetizan como:

- Identificar conflictos sociales,

- Identificar las partes de la negociación,

- Estructuración de la información personal,

- Definición de un protocolo y estrategia de negociación,

- Negociar (intercambio de ofertas, argumentación y aprendizaje),

- Renegociación.

A continuación, se presentan los aspectos metodológicos que caracterizaron este estudio. 


\section{Metodología y modelo de trabajo}

La fundamentación teórica que soporta el desarrollo metodológico de esta investigación se establece en concordancia con [16] quienes sustentan que el método analítico pretende "descomponer un todo en sus elementos constitutivos para proceder a su comprensión y rearticulación”. Centrándose en el proceso de toma de decisiones como fenómeno de estudio, se analizan y especifican las características de los escenarios de negociación, de manera que se establezcan las actividades posibles que permitan caracterizar la toma de decisiones no programadas en escenarios de negociación. El siguiente esquema (ver Fig. 2) presenta las fases de la metodología de trabajo.

\begin{tabular}{ll} 
- Fase 1 Identificación de las características de la toma de decisiones en general \\
- Fase $2 ~$ & Análisis de la toma decisiones no programadas \\
\hline - Fase 3 & Establecer la relación entre la toma de decisiones y la alta dirección \\
\hline - Fase 4 & Evaluación de las actividades o flujo de trabajo en escenarios de negociación \\
\hline Fase 5 & Desarrollo del modelo de relación entre TD no programadas y escenarios de negociación
\end{tabular}

Fig. 2. Fases de la metodología.

La siguiente sección presenta el modelo propuesto en esta investigación, en el cual se relacionan las actividades que permitirán caracterizar los procesos de toma de decisiones no programadas en escenarios de negociación.

\subsection{Modelo de relación entre TD no programadas y negociación}

El modelo aquí propuesto establece en primer lugar 4 componentes básicos de los escenarios de negociación. En segundo lugar, 3 actividades que permitirán caracterizar los procesos de toma de decisiones no programadas. Y por último, se establece una relación entre dichos elementos (escenarios de negociación y actividades de TD no programadas). Posteriormente se especifican ciertos criterios matemáticos con los que se modelarán los procesos de TD No Programadas en estudios posteriores.

Inicialmente se establecieron 4 componentes básicos (dispuestos en cada uno de los cuadrantes) que constituyen los escenarios de negociación. Estos son:

A. Contexto, problema y objetivos: Primeros pasos dentro de un proceso de negociación. Se establecen características del problema, contexto y objetivo a conseguir.

B. Partes de la negociación: Incluye establecer los oponentes, los posibles coequiperos y los asuntos bajo los cuales hay que llegar a acuerdos.

C. Protocolos y estrategias: Siendo el núcleo de la negociación, abarca las fases de diseño, planeación y ejecución de protocolos y estrategias de negociación.

D. Adaptación y reestructuración: Enmarca acciones realizadas durante la negociación y las posibles reestructuraciones si los desenlaces no toman el curso esperado. 
Por cada cuadrante se trazan las actividades que son susceptibles de ser desarrolladas con la finalidad de atender los propósitos de cada componente y con lo cual se caracterizarán los procesos de toma de decisiones no programadas. Es pertinente acotar que esto se logrará en la medida en que se involucren aspectos de ambigüedad en la información y novedad en las características del caso a enfrentar.

En ese sentido, las actividades que permitirán caracterizar los procesos de toma de decisiones no programadas que se consolidaron son (ver Fig. 3):

1. Planteamiento de alternativas: Visto como el manejo de probabilidades, procesamiento de información y manejo del tiempo para buscar y establecer opciones diferentes de actuar. Este tipo de actividades serán propuestas para atender los propósitos de los componentes A, C y D.

2. Captura de información: Entendido como aquellos procesos de percepción, de advertir y comprender características del escenario, del contexto, del problema, de los oponentes, incluso consiguiendo perfilarlos. En componentes A, B y D.

3. Relaciones personales: Abarcan aspectos de interrelaciones, procesos comunicativos, trabajo en equipo, trabajo bajo presión, concentración, habilidades para persuadir, imponer o de permeabilidad en las ideas (que tanto se deja sugestionar una persona). En componentes B, C y D.

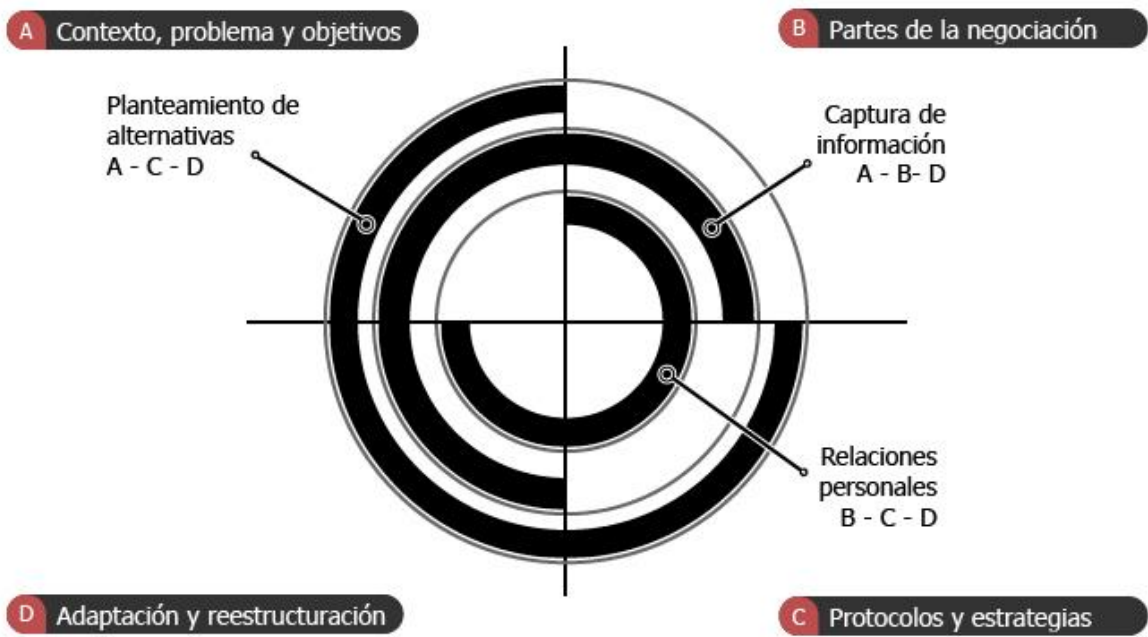

Fig. 3. Modelo de actividades para la caracterización de procesos de toma de decisiones no programadas distribuidas en los componentes de negociación.

La propuesta de este modelo también incluye la relación entre las actividades de Toma de Decisiones No Programadas y las Estrategias de Toma de Decisiones presentadas en [4], incluyendo los modelos matemáticos abordados en [5]. Esta triple relación (actividades - estrategias - criterios) representa la vía o criterio matemático por medio del que se modelaran las actividades de Toma de Decisión No Programadas en los escenarios de negociación posteriormente, haciendo uso de agentes inteligentes en entornos de gamificación. Las estrategias que no aparecen descritas por algún criterio matemático, implica un modelamiento basado en la interacción del usuario en el entorno gamificado, por ejemplo, la estrategia de "Ensayo y error", o la 
Modelo de actividades para la caracterización de procesos de toma de decisiones no programadas ...

"Experimental" podrán ponerse a prueba por el número de elecciones repetidas que tenga un usuario. Por su parte, "Costo mínimo" y "Máximo beneficio" dependerán de la forma en que se entregue la información al usuario. El resultado de este análisis es mostrado en la Tabla 1:

Tabla 1. Relación entre las Actividades de TD No Programadas y las Estrategias.

\begin{tabular}{|c|c|}
\hline $\begin{array}{l}\text { Actividades de TD } \\
\text { No Programadas }\end{array}$ & Estrategia de TD: modelo matemático (si aplica) \\
\hline \multirow{4}{*}{$\begin{array}{l}\text { 1- Planteamiento de } \\
\text { alternativas }\end{array}$} & Ensayo y error, Experimental, Experiencia \\
\hline & Certeza (probabilidades): $\sum P\left(A_{i}\right)=\sum \frac{1}{m}\left(A_{i}\right)$, para todo $i=1,2,3 \ldots m$ \\
\hline & Pesimista: $d=\operatorname{Max}\left(\operatorname{Min}_{i j}\right)=\operatorname{Max}\left(m_{i}\right)$ \\
\hline & Optimista: $d=\operatorname{Max}\left(\operatorname{Max} r_{i j}\right)=\operatorname{Max}\left(M_{i}\right)$ \\
\hline $\begin{array}{l}\text { 2- Captura de } \\
\text { información }\end{array}$ & Costo mínimo, Máximo beneficio \\
\hline $\begin{array}{l}\text { 3- Relaciones } \\
\text { personales }\end{array}$ & Arbitraria, Preferencial, Sentido Común \\
\hline
\end{tabular}

\section{Conclusiones}

Es evidente que no habrá una regla general para afrontar escenarios de negociación, sin embargo si es posible abstraer ciertas acciones y atributos con los cuales se logra dar cierta connotación especial a un proceso de negociación y que de una u otra forma pueden llegar a asegurar un idóneo abordaje por parte de un negociador. Sin embargo, el estudio de la relación entre escenarios de negociación y los procesos de toma de decisiones abordados arrojó como resultado 4 grandes categorías de actividades, sintetizando fases y renombrando algunas de las que fueron presentadas anteriormente. Donde se hacen explícitos los objetivos de las actividades a realizar y con lo cual se parametriza inicialmente el modelo basado en gamificación. Estas actividades deben apuntar a: i) identificar el contexto, problema y los objetivos a perseguir, ii) identificar el problemas y las partes de la negociación (coequiperos, oponentes y asuntos), iii) diseñar e implementar protocolos y estrategias de negociación y iv) replantear los protocolos y estrategias de negociación.

Los criterios matemáticos relacionados con las actividades de TD No Programadas a realizar, deben permitir la medición o dar indicadores, de las capacidades a ser demostradas por el negociador quien tomará las respectivas decisiones.

En detalle, las actividades se consolidaron en: i) el planteamiento de alternativas, ii) la captura de información, y iii) las relaciones personales. Esto, basado en premisas como que la toma de decisiones y el conocimiento son considerados la consecuencia final de un proceso de percepción, tratamiento y el almacenamiento de la información tal como se argumenta en [17]. Los procesos comunicativos (dispuestos dentro de la actividad de relaciones personales) tienen gran trascendencia para un efectivo curso de la negociación, en este sentido [18] logran sintetizar el beneficio que tienen la persuasión, el debate y la reflexión como actos para manejar el conflicto al decidir el curso de una acción. 
Como trabajo futuro se plantea la consolidación de un modelo de caracterización de los procesos de toma de decisiones no programadas por medio de gamificación y agentes inteligentes, para ello se abordará una revisión sistemática minuciosa y actualizada para dar respuesta a problemas encontrados en este tipo de escenarios.

\section{Referencias}

1. Bolloju, N.: Discovering classes of decision models: a step towards discovery of decision making styles, in System Sciences. Proceedings of the Thirty-First Hawaii International Conference (1998)

2. Rodriguez, E., Pedraja, L.: Análise do impacto do processo de toma da de decisoes estratégicas sobre a eficácia das organizaçoes públicas. Revista Innovar Journal Revista de Ciencias Administrativas y Sociales, Vol. 19, No. 35, 33-46 2248-6968 0121-5051 (2012)

3. Zachary, W., Wherry, R., Glenn, F., Hopson, J.: Decision situations, decision processes, and decision functions: Towards a theory-based framework for decision-aid design. Presented at the Proceedings Conference on Human Factors in Computing Systems, Gaithersburg, Maryland, USA (1982)

4. Wang, Y., Dong, L., Ruhe, G.: Formal description of the cognitive process of decision making. Proceedings of the Third IEEE International Conference on Cognitive Informatics, pp. 124-130 (2004)

5. Jiménez, L., Jiménez, M.: Algunos modelos de toma de decisiones. NOVUM: Revista de Ciencias Sociales Aplicadas, núm. 2: Novum N 2 - Revista de Ciencias Sociales Aplicadas; 33 - 48 2357-4933 0121-5698 (2014)

6. . Corona, L.A.: La teoría general sobre la toma de decisiones y su aplicación al campo de la asistencia médica (III). Medisur: Revista Electrónica de las Ciencias Médicas en Cienfuegos, Vol. 2, pp. 35-40 (2004)

7. Sarne, D., Elmalech, A., Grosz, B.J., Geva, M.: Less is more: restructuring decisions to improve agent search. International Conference on Autonomous Agents and Multiagent Systems - Volume 1, Taipei, Taiwan (2011)

8. Koontz, H., Weihrich, H., González, E.M.: Administración: una perspectiva global. McGraw-Hill Bogotá (1998)

9. Wang, Y., Patel, S., Patel, D., Ying, W.: A layered reference model of the brain. Cognitive Informatics, Proceedings, The Second IEEE International Conference on, pp. 7-17 (2003)

10. Rodrigues, S.A., de Souza, J.M.: A Web Tool to Analyse Negotiation Behavior. Multimedia and Ubiquitous Engineering (MUE), 5th FTRA International Conference on, pp. 183-188 (2011)

11. Hitt, M.A., Collins, J.D.: Business ethics, strategic decision making, and firm performance. Business Horizons, Vol. 50, pp. 353-357 (2007)

12. Plata, J.: Los "juegos gerenciales": el presente de la gerencia. Econografos, Escuela de Administración de Empresas y Contaduría Pública, pp. 1-14 (2008)

13. Pruitt, D.G.: Negotiation behavior. New York: Academic Press, Inc. (2013)

14. Fisher, R., Ury, W., Patton, B.: Das Harvard-Konzept: der Klassiker der Verhandlungstechnik: Campus Verlag (2013)

15. Lopes, F., Wooldridge, M., Novais, A.Q.: Negotiation among autonomous computational agents: principles, analysis and challenges. Artificial Intelligence Review, Vol. 29, pp. 1-44, (2008)

16. Lopera, J.D., Ramírez, C., Zuluaga, M., Vanegas, J.: El método analítico como método natural. Nómadas, Revista Crítica de Ciencias Sociales y Jurídicas, Vol. 25 (2010)

17. Bayani Abbasy, M.: Influence of simulation and real implementation Skills on the cognitive learning aspects. Cognitive Infocommunications (CogInfoCom), IEEE 3rd International Conference on, pp. 719-724 (2012) 
Modelo de actividades para la caracterización de procesos de toma de decisiones no programadas ...

18. McBurney, P., Hitchcock, D., Parsons, S.: The eightfold way of deliberation dialogue. International Journal of Intelligent Systems, Vol. 22, pp. 95-132 (2007) 